\title{
Mechanochemical modification of the composition and structure of plant raw materials to control the combustion of alternative fuel
}

\author{
Aleksey Bychkov $^{1 *}$, Ekaterina Podgorbunskikh ${ }^{1}$, Tatiana Skripkina ${ }^{1}$, Anatoly Burdukov $^{2}$, \\ and Oleg Lomovsky ${ }^{1}$ \\ ${ }^{1}$ Institute of Solid State Chemistry and Mechanochemistry, SB RAS, 630128 Novosibirsk, 18 \\ Kutateladze Str., Russia \\ ${ }^{2}$ Kutateladze Institute of Thermophysics, SB RAS, 630090 Novosibirsk, 1 Lavrentyev ave., Russia
}

\begin{abstract}
The possibilities of mechanochemistry in processing of renewable lignocellulose raw material into solid kinds of biofuel are demonstrated in this work. A review of lignocellulose raw materials promising for our country is presented. These raw materials include wastes from agriculture and forestry, and the biomass of rapidly growing plants. The physicochemical properties of lignocellulose materials with different delignification degrees were modeled with the help of the artificial mixtures of plant raw material with purified cellulose and lignin. The data illustrating the effect of disperse state and lignin content on the reactivity of the material in subsequent combustion are presented. The tests at the combustion bench with the thermal power up to 5 MW allowed determining the optimal combustion parameters for the obtained biofuel in the autothermal mode.
\end{abstract}

\section{Introduction}

Much attention in the world is paid to the shift from mineral to renewable energy sources. For example, Russia has set the ambitious goal of achieving $4.5 \%$ of renewables in 2020 , and by 2030 it is planned to provide the renewable energy at least at 80-100 billion kilowatt hours per year [1]. Significant progress has been made in the industrial production of woodbased solid biofuels and in the consumption of new kinds of biomass as raw materials [2-3].

The sources of biomasses suitable for biorefinary and biofuel production are under investigation in many developing regions of the world [4]. These sources include the biomass of plants growing in a specific region, and agricultural wastes [5]. Studies are aimed at optimization of the combustion of artificial mixtures, including lignin from black liquor and various kinds of coal [6].

The urgency of the development of technologies for the treatment of lignified wastes is defined by the fact that each kilogram of produced cereals brings up to $5 \mathrm{~kg}$ of improperly

*Corresponding author: bychkov.a.1.@gmail.com 
used or field-burnt lignocellulose wastes. One of the major biomass components is lignin; it is a polyphenol compound, and it is ballast from the viewpoint of food chemistry. At the same time, lignin has a heating value higher than that of carbohydrates, so it could be efficiently used in power engineering. However, the existing technology of lignin separation from plant raw materials (mainly by means of sulfite pulping) causes contamination with sulfur in the amount multiply exceeding the standards admissible for fuel.

The goal of the present work was mechanochemical modification of the composition and structure of plant raw material to control the combustion of fine solid biofuel.

\section{Materials and Methods}

Plant raw material annually renewable in the Russian Federation in large amounts and concentrated in the sites of growing or processing was used in the work. Wheat, oats, corn, and barley straw, biomass of reed, sedge, corncobs, originated from different districts of Novosibirsk Region. Husk of rice, pumpkin and sunflower seed samples were collected at the processing plants of Krasnodar Region. Pine bark and branches, birch branches were collected at the wood processing plant in Kurtamysh, Kurgan Region. Buckthorn branches were collected at the plantations in the Altay Region. Husk of pine nuts and hulls of pine strobiles were collected in the Tomsk Region.

The artificial model samples of biofuel with lignin content of $10-80 \%$ were prepared by mixing the weighted portions of wheat straw with the calculated amounts of pure cellulose (Sigma Aldrich, CAS \# 9004-34-6) and lignin (Sigma Aldrich, CAS \# 8068-05-1). To average the composition, thorough mixing and grinding in agate mortar was carried out for 5 minutes. The composition of wheat straw determined according to the procedure described in [7] was: lignin $-18.3 \%$, cellulose $-32.9 \%$, hemicellulose $-33.6 \%$, extractives $-9.4 \%$, and moisture $-5.9 \%$. The samples with lignin content from 0 to $100 \%$ did not contain wheat straw and were $\alpha$-cellulose and lignin, respectively.

Moisture and ash content of the samples were determined using the standard procedures [8] and [9], respectively.

Carbon, hydrogen, nitrogen and sulfur content was determined with the help of an automatic analyzer EURO EA(Italy) according to procedures described in [10]. Higher heating values (HHV) were determined according to the procedure described in [11].

\section{Results and Discussion}

The wastes from agriculture and forestry were chosen as the promising lignocellulose raw material for obtaining the solid biofuel. The main criterion for the choice was the formation and/or accumulation of large amounts of the chosen kinds of biomass. We also considered wild plants that form localized resources in nature. Fuel with different content of hydrolytic lignin was modeled with the help of artificial mixtures of wheat straw (the basic component) and the calculated amounts of pure cellulose or lignin.

The results of the elemental analysis and determination of the ash content [12] of samples show that an increase in lignin content leads to a substantial increase in the content of the major element - carbon. At the same time, an increase in the ash content of the lignocellulose material is accompanied by the depletion of its organic composition, which must have a negative effect on the heating value. The highest ash content is that exhibited by rice husk; it may contain up to $20 \%$ amorphous silicon dioxide in its supramolecular structure. The minimal ash content is that of the husk of pine nuts (less than $1 \%$ ), which 
allows one to obtain low-ash solid fuel from this kind of raw material, in particular fuel pellets of the Premium grade.

The maximal combustion heat is produced by sunflower seed husk; this value is close to those of highly lignified model samples. This fact for the natural raw material can be explained by the features of its composition. For example, sunflower seed husk contains a definite amount of lipids remaining after the removal of seeds; these lipids are characterized by high combustion heat. High ash content of the material (rice husk and oat straw) or low lignin content (eg. corncobs) leads to a substantial decrease in the heat of combustion.

From the modern point of view, the combustion efficiency of fine fuel is affected to a higher extent by two major factors: combustion rate, connected with particle size, and activation energy, connected with the chemical composition of fuel. In the present work, the kinetics of oxidation of fine fuel samples characterized by different dispersity and different lignin content was studied using the methods of thermal analysis. Wheat straw was used as a raw material to prepare the samples.
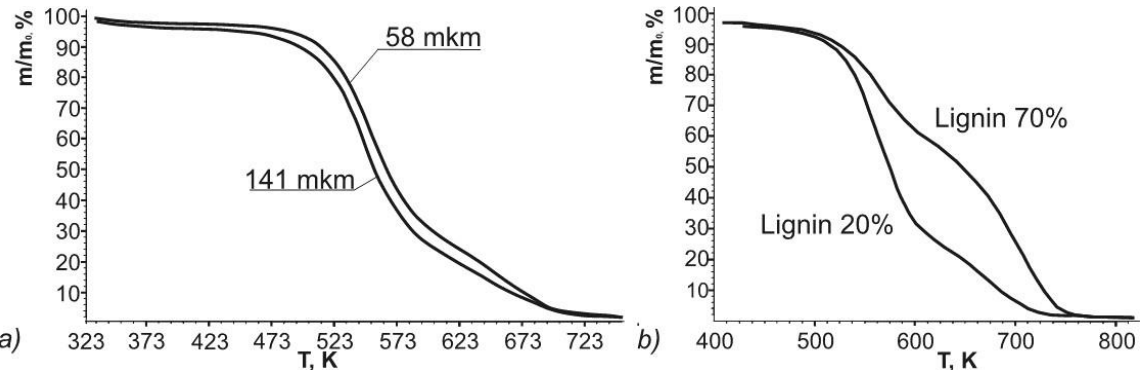

Fig. 1. Curves of oxidation of lignocellulose fuel samples: $a$ - with different particle sizes, $b$ - with different lignin content. Heating rate: $10 \mathrm{~K} / \mathrm{min}$.

One can see from the shapes of the curves of biofuel combustion that the nature of oxidation process does not change principally depending on dispersity (Fig. 1, a). In both cases (average particle sizes in the samples were 58 and $141 \mathrm{mkm}$, respectively) the major mass loss is observed at 500-575 K with a small shoulder at 590-675 K. According to the modern data [13], this situation corresponds to the reactions of hemicellulose and cellulose combustion (the reaction of lignin combustion is not revealed separately by means of thermogravimetry; instead, it forms a broad halo on the mass loss curve within temperature range 520-770 K). The rate (the slope of the tangent to the abscissa axis) for the sample with particle size $58 \mathrm{mkm}$ is approximately equal to that for the sample with particle size $141 \mathrm{mkm}$. This allows us to suppose that particle size within the studied range is not a limiting factor for combustion rate. This conclusion is extremely important for the economics of the production of dispersed lignocellulose fuel because mechanochemical grinding of plant raw material to obtain particles smaller than $150 \mathrm{mkm}$ requires much energy.

The curves of mass loss (Fig. 1, $b$ ) illustrate oxidation of the samples of fine fuel with lignin content of 20 and $70 \%$, respectively. One can see that an increase in lignin content in fuel changes the character of oxidation process. For instance, for the sample with lignin content of $20 \%$, the kinetics presented in Fig. 1 is characteristic. At the same time, for the sample with lignin content of $70 \%$, the major mass loss occurs at the high-temperature stage, which is likely the evidence of a shift of the contribution from lignin halo to the hightemperature region. It is logical to assume that lignin combustion at high temperature advantageously distinguishes highly lignified fuel samples from low-lignin samples. In addition, the rate of oxidation at the high-temperature stage of the process increases by $7 \%$.

These changes in combustion rates lead us to the conclusion that the combustion of hemicellulose and cellulose may become the limiting stage in a highly lignified sample, 
while lignin gets burnt rather rapidly in the high-temperature region. In this connection, it is necessary to choose the conditions of fuel combustion so that the particles could be heated as soon as possible to the high-temperature range, so that the heating value of lignin could be used in full for the afterburning of carbohydrate polymers.

Investigation of the parameters of lightning, inflammation, and stable autothermal combustion of biofuel with different dispersity and composition was carried out at the combustion bench with the thermal powder up to $5 \mathrm{MW}$. These tests allowed us to reveal acceptable thermodynamic parameters of combustion (temperature, the ratio of fuel under investigation to the oxidizer at the first and second stages of the burner) meeting the standardized ecological characteristics of combustion [14]. The optimal technological parameters are: the excess of primary air with respect to the theoretical value for complete combustion $0.25-0.3$, the ratio of primary to secondary air 1.2-1.4.

Thus, the possibilities of mechanochemical methods in obtaining fine solid biofuel from renewable raw material are demonstrated in the work. We carried out the screening studies of raw materials promising for Russian Federation, namely the wastes of agriculture and forestry, and the biomass of rapidly growing plants. It is demonstrated that the nature of oxidation process does not change principally with the decrease in average particle size from $141 \mathrm{mkm}$ to $58 \mathrm{mkm}$. In all cases, the major mass loss is observed at 500-575 K, with a small contribution form the second stage at 590-675 K. It is demonstrated with the help of artificial samples with different composition that an increase in the fraction of lignin leads to a substantial increase in the heat of combustion and to a shift of oxidation process into the high-temperature region.

This work was supported by the Russian Science Foundation (project no. 16-13-10200)

\section{References}

1. Roadmap EU-Russia Energy Cooperation until 2050. March 2013. https://ec.europa.eu/energy/sites/ener/files/documents/2013_03_eu_russia_roadmap_20 50_signed.pdf Accessed 29 Mar 2017.

2. S. Proskurina, J. Heinimö, M. Mikkilä, E. Vakkilainen, Renew. Sust. Energ. Rev. 51, 730 (2015).

3. P. Maryandyshev, A. Chernov, V. Lyubov, G. Trouve, A. Brillard, J-F. Brilhac, J. Therm. Anal. Calorim. 122, 2 (2015)

4. Y. Su, P. Zhang, Y. Su, Renew. Sust. Energ. Rev. 50, 991 (2015)

5. N. Gaurav, S. Sivasankari, G.S. Kiran, A. Ninawe, J. Selvin, Renew. Sust. Energ. Rev. 73, 205 (2017)

6. S. Mesfun, J. Lundgren, C.E. Grip, A. Toffolo, P.L.K. Nilsson, U. Rova, Bioresource Technol. 166, 508 (2014)

7. A.L. Bychkov, E.I. Ryabchikova, K.G. Korolev, O.I. Lomovsky, Biomass Bioenerg; 47, 260 (2012)

8. Standard \# EN 14774-3 "Solid biofuels - Determination of moisture content" (2009)

9. Standard \# EN 14775 "Solid biofuels - Determination of ash content" (2009)

10. V.P. Fadeeva, T.D. Tikhova, O.N. Nikulicheva, J. Anal. Chem. 63, 11 (2008)

11. ISO 1928:2009-06 "Solid mineral fuels - Determination of gross calorific value by the bomb calorimetric method and calculation of net calorific value" (2009)

12. A.L. Bychkov, A.I. Denkin, V.D. Tikhova, O.I. Lomovsky, J. Therm. Anal. Calorimetry (to be published in 2017)

13. H. Haykiri-Acma J. Anal. Pyrolysis. 75, 211-216. (2006)

14. A.P. Burdukov, M.Y. Chernetskiy, A.A. Dekterev, I.S. Anufriev, P.A. Strizhak, P.Yu. Greben'kov, Therm. Eng. 63, 1 (2016) 